\title{
Rupture risk in abdominal aortic aneurysms: A realistic assessment of the explicit GPU approach
}

\author{
V. Strbac ，D.M. Pierce ，B. Rodriguez-Vila , J. Vander Sloten , N. Famaey
}

\begin{abstract}
A B S T R A C T
Accurate estimation of peak wall stress (PWS) is the crux of biomechanically motivated rupture risk assessment for abdominal aortic aneurysms aimed to improve clinical outcomes. Such assessments often use the finite element (FE) method to obtain PWS, albeit at a high computational cost, motivating simplifications in material or element formulations. These simplifications, while useful, come at a cost of reliability and accuracy. We achieve research-standard accuracy and maintain clinically applicable speeds by using novel computational technologies. We present a solution using our custom finite element code based on graphics processing unit (GPU) technology that is able to account for added complexities involved with more physiologically relevant solutions, e.g. strong anisotropy and heterogeneity. We present solutions up to $17 \times$ faster relative to an established finite element code using state-of-the-art nonlinear, anisotropic and nearly-incompressible material descriptions. We show a realistic assessment of the explicit GPU FE approach by using complex problem geometry, biofidelic material law, doubleprecision floating point computation and full element integration. Due to the increased solution speed without loss of accuracy, shown on five clinical cases of abdominal aortic aneurysms, the method shows promise for clinical use in determining rupture risk of abdominal aortic aneurysms.
\end{abstract}

\section{Introduction}

\subsection{Abdominal aortic aneurysms}

An Abdominal Aortic Aneurysm (AAA) is a permanent enlargement (more than $50 \%$ ) of the abdominal aorta and is often referred to as "the silent killer" due to its inconspicuous onset and growth. Once ruptured, reported mortality rates are as high as $90 \%$ (Fleming et al., 2005), making it the 10th leading cause of death for men over the age of 65 (Vardulaki et al., 1998). AAAs are usually detected by ultrasound screening, an effective and economical approach. Subsequent Computed Tomography (CT) scans are an important means of gathering information and decision-making for elective surgery - the primary treatment method. Current clinical practice predominantly relies on statistically motivated and simplistic geometric indicators in decision-making for elective repair: maximum diameter $>5.4 \mathrm{~cm}$ or $\geqslant 1 \mathrm{~cm}$ diameter yearly growth (Johansson et al., 1990; Chaikof et al., 2009).

\subsection{Biomechanically motivated rupture risk assessment}

Over the last decade, researchers pursued more sophisticated, biomechanically motivated indicators for rupture risk assessment, showing that Peak Wall Stress (PWS) correlates better with clinically observed outcomes than the aforementioned geometric criteria (Fillinger et al., 2002; Martufi et al., 2016). Increased accuracy of stress estimations require biofidelic continuum mechanics-based descriptions of aortic tissue (Pierce et al., 2015b; Gasser et al., 2006). Proper spatial discretization of the aortic domain in question, appropriate material descriptions and prescribed conditions on the boundaries define a discretized boundary value problem (BVP) in elasticity. The finite element (FE) method is a conventional and well-established tool to solve the BVP and obtains the resulting stress and deformation field throughout the aorta.

\subsection{FE analysis}

\subsubsection{FE analysis: basics of computation and efficiency}

FE methods can be classified into explicit and implicit methods depending on the numerical integration scheme used. The suitability of each approach varies with the nature of the problem. One 
commonly solves quasi-static problems implicitly, but regardless of the method, FE analysis is computationally demanding and thus relatively slow. Computational tasks whose constituents are sequentially independent (exhibit internal parallelism) are particularly well-suited for parallelization - an essential tool in decreasing solution time. Implicit solutions involve the assembly and solution of a large (typically sparse) system of equations, which is avoided in explicit solutions. Explicit solvers generally decouple systems of equations, making them more fit to parallelization. While setting up simulations with either method requires proper care and awareness, when using explicit $\mathrm{FE}$, one needs to account for damping as well as additional constraints related to mesh quality affecting the time-step size.

\subsubsection{Meshing and mechanical model of AAAs for FE analysis}

The aorta has a complex geometry given its bifurcation, branching arteries, its layered wall structure and potential presence of intraluminal thrombus (ILT). Obtaining high fidelity mesh representations of the morphology is a challenging open question. Tetrahedral elements, while simpler to discretize with, are generally less reliable and efficient in resolving stresses than hexahedral elements, particularly with nonlinear and incompressible materials (Puso and Solberg, 2011).

Given a fixed aorta geometry derived from imaging, the most significant factor in obtaining good solutions is mesh density. The objective is to minimize the number of elements while resolving the stress field. A higher number of elements usually implies a better quality of elements in terms of aspect ratio and skewness, but the strategy is not applicable for all geometries. Moreover, an increased number of elements nonlinearly increases the solution times. If accounting for the layered morphology and non-uniform thickness, conformal projection-based methods in De Santis et al. (2011), Tarjuelo-Gutierrez et al. (2014), Auer and Gasser (2010) appear effective. The thickness direction should be represented with sufficient elements to represent stress gradients through the bulk of the aorta - generally 3-6 layers.

The morphology of the domain is such that the transition from the aortic wall to ILT along the axial direction of the aorta is smooth. The geometric representation of this material separation therefore necessarily contains (poor quality) collapsed "wedge" elements, regardless of the meshing algorithm or even mesh density (Tarjuelo-Gutierrez et al., 2014).

The intraluminal thrombus and variations in wall thickness are essential parameters influencing the rupture risk of AAAs (Gasser et al., 2010). The mechanical influence of ILT is different for every patient, focusing attention to its structure and accurate geometric and mechanical representation. The current clinical imaging modality of choice (CT) does not allow differentiation between the ILT and vessel wall border, so previous publications define different approximate methods to obtain vessel wall thickness (De Santis et al., 2011; Auer and Gasser, 2010). However, Magnetic Resonance Imaging (MRI) technology can discriminate ILT from aortic tissue, and allows including accurate patient-specific ILT morphology in FE simulations.

The complex mechanical response of the aorta is nonlinear, anisotropic, heterogeneous and nearly-incompressible (Holzapfel et al., 2000). Continuum-mechanical models that reflect the histology of the aorta as a composite of an isotropic soft ground matrix and an embedded anisotropic distributed network of collagen fibers are common (Gasser et al., 2006; Holzapfel et al., 2005).

\subsubsection{Undeformed configuration}

The in vivo captured (imaged) morphology of patients' anatomy is both prestressed (loaded) and residually stressed, and should not be used as a stress-free "initial" configuration in FE simulation. Several methods attempt to ascertain an internal stress distribu- tion in equilibrium with the known boundary conditions (de Putter et al., 2007; Bols et al., 2013; Weisbecker et al., 2014; Joldes et al., 2015; Pierce et al., 2015a), and to account for (still present!) residual stresses (Pierce et al., 2015a; Bellini et al., 2014; Alastrué et al., 2007; Alastrué et al., 2010). There is currently no panacea for quick and reliable determination of the stress-free configuration, and most methods rely on a series of forward simulations, increasing overall computational cost.

\subsection{GPU-accelerated explicit FE analysis}

Graphics processing unit (GPU) technology is proposed for medical image segmentation (Smistad et al., 2015), or deformable models using spring-mass-damper or tensor-mass methods (Rasmusson et al., 2008) as well as implicit FE (Cecka et al., 2011; Wong et al., 2015). The Total Lagrangian Explicit Dynamic FE algorithm (TLED) (Miller et al., 2007) fits the GPU programming model well given: (1) most element and nodal operations are pleasingly parallel (independent of one another), and (2) memory requirements are very low.

Reports of large (up to two orders of magnitude) speed-ups can be found in the literature for the TLED method Taylor et al. (2009), Strbac et al. (2015), Strbac et al. (2016), Bartezzaghi et al. (2015). Such claims are often based on simplifications, some introduced in the following paragraphs. While useful in specific scenarios, highly accurate simulation sought here precludes these simplifications.

Initial publications on the topic (Comas et al., 2008; Joldes et al., 2009) do not utilize double-precision (fp64) computation, as Nvidia GPUs did not support it. With the release of Compute Capability 1.3 devices in late 2008 , fp64 computation is supported, but is still largely absent in literature. Bartezzaghi et al. (2015) first addressed this issue, and with the exception of Strbac et al. (2015) and Strbac et al. (2016), the authors are unaware of reports using doubleprecision computation in our context. It is well-known that double-precision computation, particularly in atomic operations, is significantly slower but mandatory in accurate and general engineering application. Huthwaite (2014) also presents fp64 results, albeit in an unrelated context.

Similarly, reports utilizing complex anisotropic material models are rare in GPU-supported explicit FE. The only exception is the group of Taylor et al. (2009), Han et al. (2010), Han et al. (2012) who investigate simpler transversely isotropic and orthotropic materials, albeit in single-precision (fp32). Regardless, accurate representations of many tissues, including aortic tissue, require complex distributed fiber-reinforced anisotropic models.

Further, benchmarking reports on GPU performance use simple or ideal (Bartezzaghi et al., 2015; Comas et al., 2008; Strbac et al., 2015) geometries. Meshing of complex geometries often requires misshaped elements (that limit usable step sizes in explicit analysis), also detailed further in Section 2.3.1.

Despite their parallelizability, a critical weakness of all explicit methods lies in the level of usable incompressibility. In soft tissues, high bulk moduli in particular limit the applicable time step, with obvious impact on solution times. Often, publications utilize lessthan-strict adherence to near-incompressibility (Joldes et al., 2010), with Poisson ratios closer to $v=0.49$, only loosely sufficient for accurate analyses, especially involving anisotropic materials that comprise largely of water, as shown by e.g. Han et al. (2012).

Strong anisotropic behavior and collapsed elements often preclude the use of element under-integration (UI). Instead, for reasons of accuracy and reliability, the fully-integrated (FI, $2 \times 2 \times 2$ integration points) quadrature scheme is advised, carrying a significant performance impact. With the exception of Strbac et al. (2016), we are unaware of other reports that use full or selectively-reduced integration (SRI) in this context. In conclusion, 
for GPU-supported explicit FE, using double-precision, nearlyincompressible anisotropic materials, full quadrature, in the presence of degenerated hexahedral elements is unreported in literature (see Appendix A, and constitutes a complex and realistic scenario.

As TLED is often reported and assessed for real-time or nearreal-time application, we examine the extensibility of the reported TLED implementations to complex, clinically applicable cases. We use conformal meshes for ILT and aortic wall, and provide a detailed analysis (typically performed in pre-processing) of representative examples of models adequate for patient-specific AAA modeling. We validate results against an established (implicit) FE code FEAP (University of California, Berkeley). We show that even in the presence of stringent detrimental factors - excellent and clinically applicable solution speeds are achievable.

\section{Materials and methods}

\subsection{Explicit FE method}

We created a custom implementation of the Total Langragian Explicit Dynamic formulation for GPU computation using CUDA (Nvidia Corp., 2015), the details of which can be found in Strbac et al. (2015). Briefly, we obtain stresses and strains through the solution of the standard second-order nonlinear system

$[\mathbf{M}]\{\ddot{\mathbf{u}}\}+q[\mathbf{M}]\{\dot{\mathbf{u}}\}+\left\{\mathbf{f}^{\text {int }}(\{\mathbf{u}\})\right\}=\left\{\mathbf{f}^{\text {ext }}\right\}$,

where $\{\mathbf{u}\}$ is the displacement vector, $[\mathbf{M}]$ is the mass matrix, $q$ is the damping coefficient, and $\left\{\mathbf{f}^{\mathrm{int}}\right\}$ and $\left\{\mathbf{f}^{\mathrm{ext}}\right\}$ are vectors of internal and external forces, respectively.

We solve the equation in time using an explicit constant step central differences operator. Given the dynamic nature of the system, we include damping to obtain a static solution (Strbac et al., 2015; Joldes et al., 2011). We use both diagonal mass and damping matrices. A diagonalized (lumped) matrices decouple the system of equations, thereby eliminating matrix inversion. Additionally, it allows processing of both element (determination of stress, integration) and nodal (time-marching) operations to be independent, hence pleasingly parallel.

\subsection{Material models}

We use convex strain energy density functions (SEDFs) to describe the continuum mechanics of aortic and thrombus tissue, defined as

$\Psi=\mathrm{U}(J)+\bar{\Psi}_{\mathrm{gm}}\left(\bar{I}_{1}\right)+\bar{\Psi}_{\mathrm{fib}}^{f}\left(\bar{I}_{1}, \bar{I}_{4}, \bar{I}_{6}\right)$,

where $f \in\{\mathrm{a}, \mathrm{t}\}$ for the aorta and ILT respectively, $\bar{\Psi}_{\mathrm{gm}}$ denotes the isotropic response of the ground matrix $\Psi_{g m}=\mu\left(\bar{I}_{1}-3\right) / 2, \mu$ is the shear modulus, $\bar{I}_{1}$ is the first invariant of the deviatoric Cauchy-Green deformation tensor $\overline{\mathbf{b}}=J^{-2 / 3} \mathbf{b}$ where $\mathbf{b}=\mathbf{F F}^{\mathrm{T}}, \mathbf{F}$ is the deformation gradient and $J=\operatorname{det}(\mathbf{F})$. Near-incompressibility is enforced by using a penalty function $\mathrm{U}=K(J-1)^{2} / 2$, in which the bulk modulus $K \gg \mu$ (Helfenstein et al., 2010) is selected to be at least three orders of magnitude larger than the initial (ground matrix) shear modulus and acts as the penalty parameter. The distributed network of collagen fibers contributes to the mechanical response of the aorta ( $\bar{\Psi}_{\text {fib }}^{a}$ ) as (cf. Gasser et al., 2006)

$\bar{\Psi}_{\text {fib }}^{a}=\sum_{i=4,6} \frac{k_{1}}{2 k_{2}}\left[e^{k_{2}\left(x \bar{l}_{1}+(1-3 \kappa) \bar{l}_{i}-1\right)^{2}}-1\right]$,

where $k_{1}>0$ is a stress-like parameter, $k_{2} \geqslant 0$ is a dimensionless parameter, $\bar{I}_{4}=\overline{\mathbf{b}}: \mathbf{M} \otimes \mathbf{M}$ and $\bar{I}_{6}=\mathbf{b}: \mathbf{M}^{\prime} \otimes \mathbf{M}^{\prime}$ with vectors $\mathbf{M}$ and $\mathbf{M}^{\prime}$ denoting the principal fiber directions in the reference (Lagrangian) configuration. A single parameter $\phi$ uniquely defines $\mathbf{M}$ and $\mathbf{M}^{\prime}$ through an angle between the circumferential direction of the artery and the fibers in the plane of the tissue. The parameter $\kappa \in[0,1 / 3]$ governs the fiber dispersion about the principal orientations, $\mathbf{M}$ and $\mathbf{M}^{\prime}$.

The function $\Psi_{\text {fib }}^{t}$ Holzapfel et al. (2005), Tong et al. (2011), Pierce et al. (2015a) describes the thrombus as

$\bar{\Psi}_{\mathrm{fib}}^{t}=\sum_{i=4,6} \frac{k_{1}}{2 k_{2}}\left[e^{k_{2}(1-\rho)\left(\bar{I}_{1}-3\right)^{2}+\rho\left(\overline{(}_{i}-1\right)^{2}}-1\right]$

where $\rho \in[0,1]$ (dimensionless) governs the fiber dispersion.

\subsection{Simulation workflow analysis}

\subsubsection{Pre-processing and model analysis}

The meshes comprise largely regular hexahedral elements, with few collapsed hexahedrals in areas of separation between ILT and aortic tissue (from TarjueloGutierrez et al., 2014), cf. Fig. 1. Each mesh consists of at least two element layers per material layer. The number of hexahedral elements per aorta range from 24,186 to 27,720 hexahedrals (cf. Table 1) and an additional 3106 to 3354 quadrilaterials for pressure, all fully-integrated.

To assess mesh quality, we opt for the Jacobian metric, which is defined as the minimum determinant of the Jacobian matrix, evaluated at each corner and at the center (Stimpson et al., 2007). Only elements with a positive value are valid for FE simulation.

We assign median values of $\mu, \kappa, \rho, \phi, k_{1}$ and $k_{2}$ to the intima, media and adventitia taken from nine tissue samples (Weisbecker et al., 2012). We take mean thrombus material parameters for luminal, medial and abluminal layers from Tong et al. (2011), cf. Table 2. For clarity, the material assignments on a mesh crosssection of Patient 1 (P1) are shown in Fig. 2.

The conditional stability of explicit FE warrants considerable care in choosing the appropriate time-step. We determine relevant diagnostic information on the related geometric, material and time-step information prior to or during simulation. We approximate the upper bound of the step size (via a maximum eigenvalue estimate) using the well-established Courant-Friedrichs-Lewy (CFL) condition (Courant et al., 1967; Belytschko, 1976)

$\Delta t \leqslant \min \left(\frac{L_{e}}{c_{e}}\right), \quad L_{e}=\frac{V_{e}}{A_{e}}, \quad c_{e}=\sqrt{\frac{4 / 3 \mu+K}{\rho_{m}}}$,

where $L_{e}$ is the characteristic length, $A_{e}$ and $V_{e}$ are the largest area and the volume of an element respectively, $c$ is the wave speed based on the elastic properties, and $\rho_{m}$ is the mass density always set to $1000 \mathrm{~kg} / \mathrm{m}^{3}$. Coplanarity is assumed in area calculations making them trivial, while for volume calculations we use a very efficient method described in Grandy (1997). Critical elements with small individual step sizes have crucial impact on solution time as they dictate overall usable time-step size.

\subsection{FE solution}

We apply a pressure boundary condition using a smooth polynomial curve (Strbac et al., 2015) on the inner intimal surface of the patient-specific meshes. We apply a systolic pressure of $120 \mathrm{mmHg}$ while keeping the proximal $(Z=\max (Z)$ plane $)$ and distal nodes $(Z=\min (Z)$ plane $)$ of the meshes fixed.

We choose the convergence criterion such that execution terminates when the kinetic energy of the entire system reaches a threshold value of $E_{k i n}<1 \times 10^{-9} \mathrm{~J}$, computed as

$\mathrm{E}_{\mathrm{kin}}=\sum_{n=1}^{N} \frac{1}{2}\left(\mathbf{v}_{n}^{t}\right)^{T} \mathbf{M} \mathbf{v}_{n}^{t}$,

where $\mathbf{v}$ is velocity, $\mathrm{t}$ denotes the current step, $n$ is the node number, and $\mathrm{N}$ is the total number of nodes in the model.

We run inflation simulations on all five patients and compare results and solution times between our custom CUDA TLED code (Strbac et al., 2015) and FEAP, both using the same fully-integrated solid (continuum) elements and identical material models.

For each patient, we perform accuracy tests by periodically computing the rootmean-square error (RMSE) between the known benchmark FEAP and intermediate configurations of the CUDA solution as the simulation evolves. The RMSE is sensitive to outliers in all dimensions, and implies the correctness of stresses. We compute RMSE as

RMSE $=\sqrt{\frac{\sum_{n=1}^{N}\left(\hat{\mathbf{u}}_{n}-\mathbf{u}_{n}^{t}\right)^{2}}{N}}$,

where $\hat{\mathbf{u}}$ denotes the reference solution displacements. At the final simulation step, as the convergence condition is triggered, the error is such that RMSE $\approx 0$. PWS is then the maximum principal stress in the solution. We compute $E_{\mathrm{kin}}$, RMSE and PWS periodically, on the GPU, and use a fast parallel reduction technique (cf. Harris, 2013).

We run the custom FE code on an Nvidia GTX980 GPU device, and FEAP on a Xeon (E5645 24 GB RAM, Windows OS, 64bit) workstation using a single core.

\section{Results}

\subsection{Pre-processing and model analysis}

Guided by the CFL condition, we break down the models in terms of their initial geometric properties and material stiffnesses 

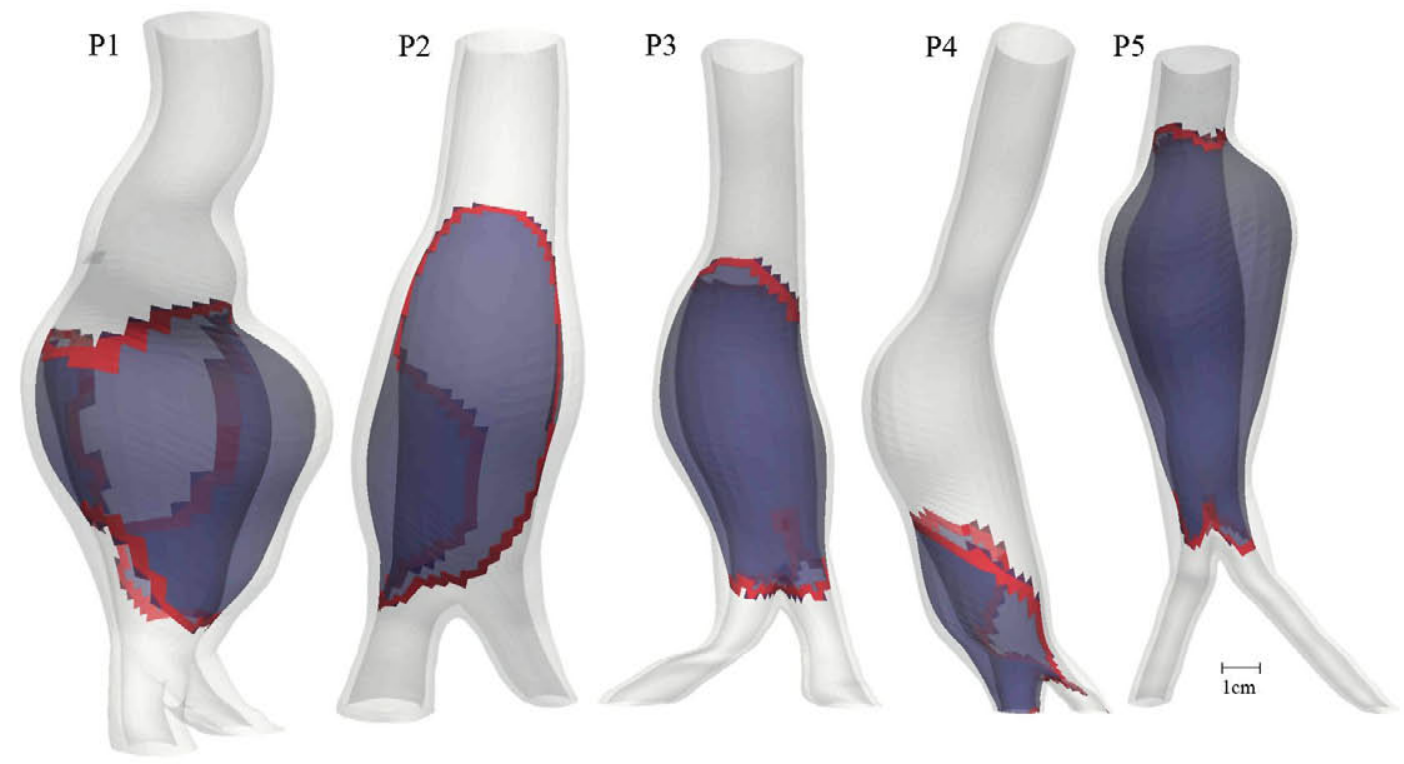

Fig. 1. FE meshes of patient-specific abdominal aortic aneurysms with highlighted ILT (blue) elements and critical (red) elements. (For interpretation of the references to color in this figure legend, the reader is referred to the web version of this article.)

Table 1

Mesh quality in terms of time-step implications. Collapsed elements are highlighted in Fig. 1.

\begin{tabular}{|c|c|c|c|c|c|c|}
\hline & Hexahedra rightarrow tal & Hexahedra collapsed & $\min \left(L_{e}\right)[\mathrm{mm}]$ & $\max \left(L_{e}\right)[\mathrm{mm}]$ & $\min (\Delta t)[s]$ & $\max (\Delta t)[\mathrm{s}]$ \\
\hline P1 & 24624 & 876 & $4.7 \times 10^{-3}$ & 3.43 & $5.9 \times 10^{-8}$ & $4.1 \times 10^{-5}$ \\
\hline P2 & 24186 & 840 & $7.5 \times 10^{-3}$ & 2.90 & $9.6 \times 10^{-8}$ & $3.4 \times 10^{-5}$ \\
\hline P3 & 24582 & 720 & $7.0 \times 10^{-3}$ & 0.96 & $8.5 \times 10^{-8}$ & $1.1 \times 10^{-5}$ \\
\hline P4 & 24888 & 834 & $5.6 \times 10^{-3}$ & 0.97 & $5.7 \times 10^{-8}$ & $1.1 \times 10^{-5}$ \\
\hline P5 & 27720 & 648 & $5.9 \times 10^{-3}$ & 3.31 & $7.6 \times 10^{-8}$ & $3.9 \times 10^{-5}$ \\
\hline
\end{tabular}

Table 2

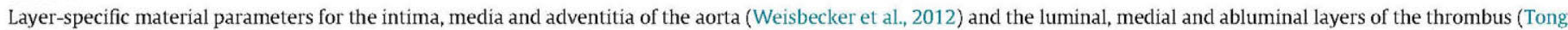
et al., 2011).

\begin{tabular}{|c|c|c|c|c|c|}
\hline & $\mu[\mathrm{MPa}]$ & $k_{1}[\mathrm{MPa}]$ & $k_{2}[-]$ & $\kappa[-]$ & $\phi\left[^{\circ}\right]$ \\
\hline \multicolumn{6}{|c|}{ Aorta } \\
\hline Intima & 0.0440 & 10.100 & 0.0 & 0.25 & 40.5 \\
\hline Media & 0.0280 & 0.8100 & 12.4 & 0.18 & 39.1 \\
\hline \multirow[t]{2}{*}{ Adventitia } & 0.0100 & 0.3800 & 3.35 & 0.11 & 40.6 \\
\hline & $\mu[\mathrm{MPa}]$ & $k_{1}[\mathrm{MPa}]$ & $k_{2}[-]$ & $\rho[-]$ & $\phi\left[^{\circ}\right]$ \\
\hline \multicolumn{6}{|c|}{ Thrombus } \\
\hline Luminal & 0.0097 & 0.0159 & 0.03 & 0.33 & 84.1 \\
\hline Medial & 0.0071 & 0.0060 & 0.07 & 0.05 & 86.7 \\
\hline Abluminal & 0.0051 & 0.0029 & 2.70 & 0.05 & 89.1 \\
\hline
\end{tabular}

(cf. Table 1) to estimate an initial step size. Critical elements coincide with collapsed hexahedrals exclusively contained in the separation zone between ILT and the intima (cf. Fig. 1). These elements do not satisfy the Jacobian quality metric with values equal to zero. To further the point, Fig. 5 shows the correlation between the Jacobian quality metric and the characteristic length $L_{e}$, and that the resulting element-wise time-steps are lowest in this region.

\subsection{GPU execution results and validation}

Variations in maximum element eigenfrequencies make the CFL step approximation in Eq. (5) conservative (Belytschko et al., 2000). Using the CFL as a guideline, we determine the largest usable step (slightly higher than predicted) and execute the simulations using the in-house developed CUDA TLED FE code. We show resulting stress fields for all patients in Fig. 3. Using our FE code, we obtain equilibrium and identical solutions within three hours, yielding speedups ranging from $10 \times$ to $17 \times$ relative to benchmark solutions. We show the initial and final RMSE values, time-step sizes, total number of steps necessary and final solution run-times in Table 3.

We illustrate the necessity of using double-precision, a high Poisson ratio and full integration in Fig. 4, with identical remaining simulation parameters. Only this combination of simulation parameters yields accurate solutions relative to the FEAP reference, avoiding either divergence, instability or incorrectness. We show the impact of deviating from these parameters in Table 4.

\section{Discussion}

We show pre-processing analysis results (cf. Section 3.1) of models without concessions to geometric or material descriptions using our custom FE code. Further, solution speeds confirm that exploiting the high parallelizability of the explicit FE method, 


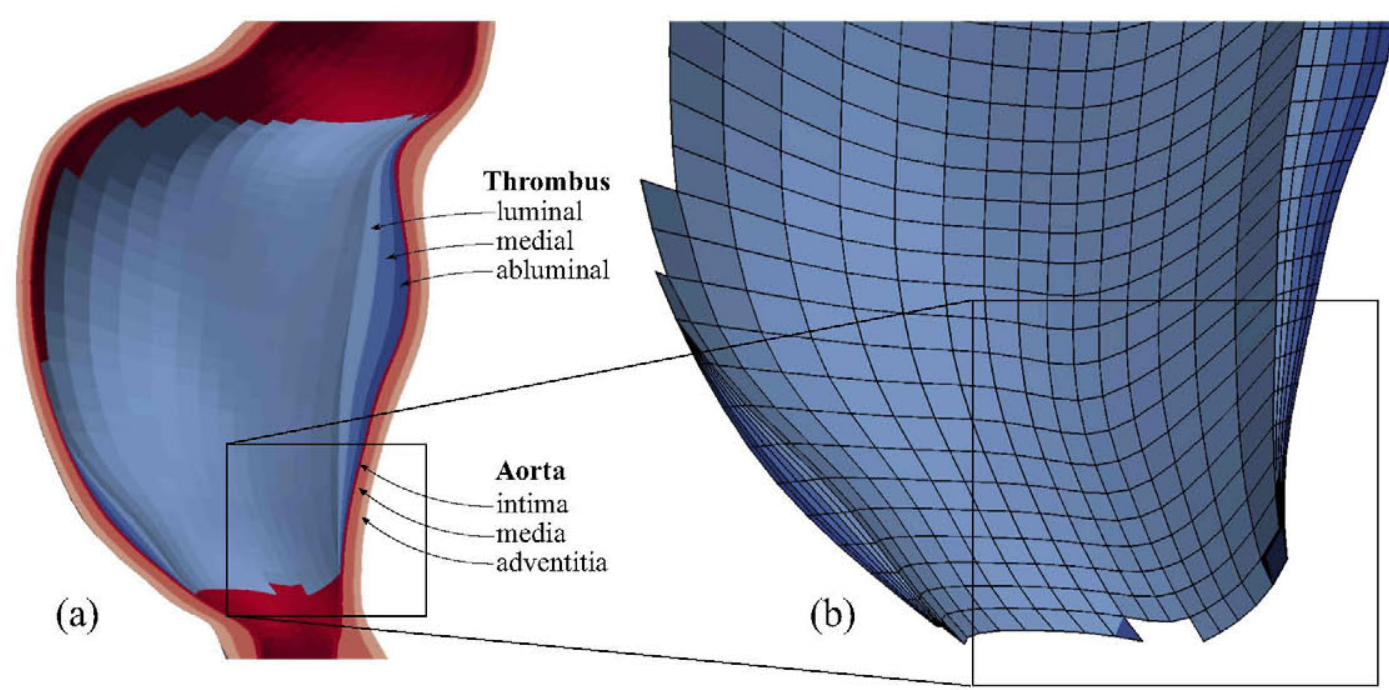

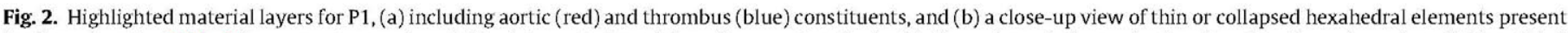

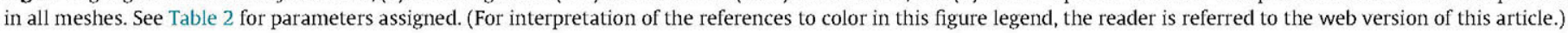
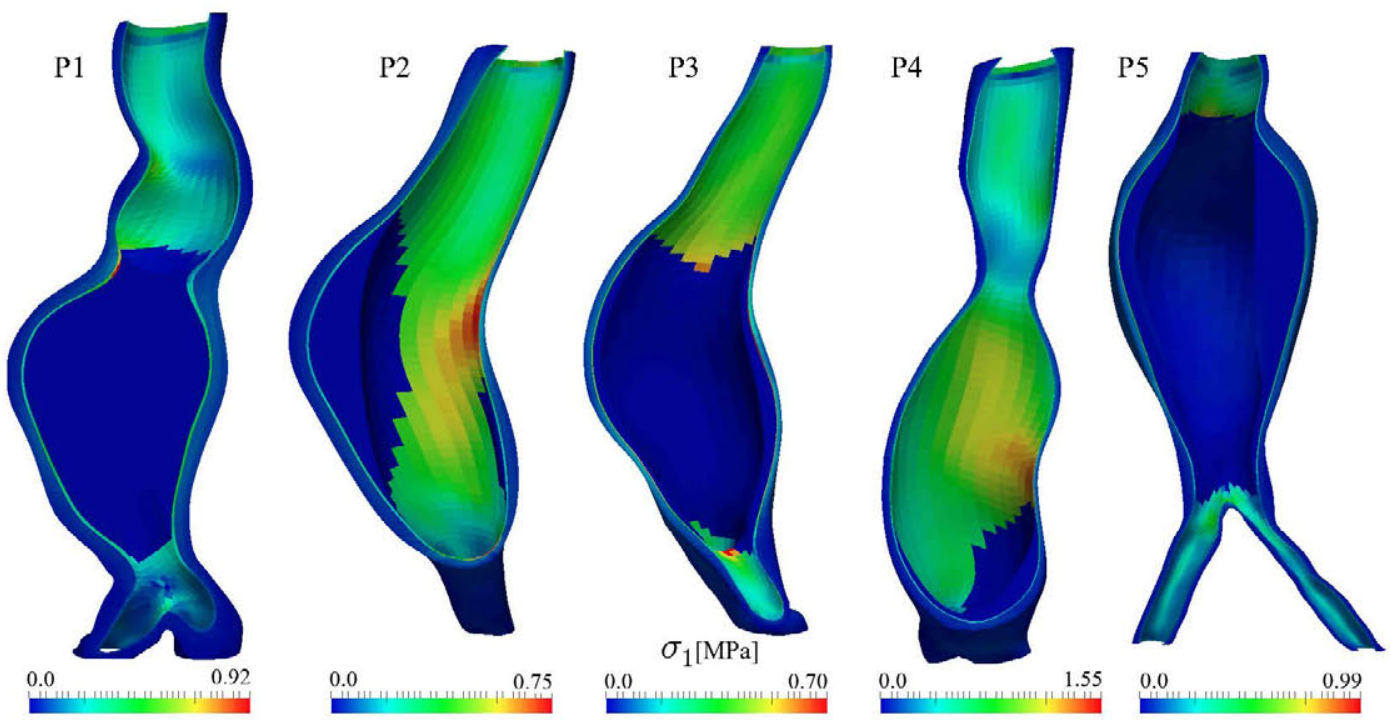

Fig. 3. Resulting first principal stresses for P1 to P5.

Table 3

Comparative results, including final root-mean-square error RMSE values, kinetic energy $\mathrm{E}_{\mathrm{kin}}$, time-steps used and solution times for our custom CUDA FE code and FEAP.

\begin{tabular}{|c|c|c|c|c|c|c|c|}
\hline & RMSE start [mm] & RMSE end [mm] & $\mathrm{E}_{\mathrm{kin}}\left[10^{-3} \mathrm{~J}\right]$ & Step size [s] & Total steps & FEAP run-time [h] & CUDA run-time [h] \\
\hline P1 & 2.0119 & $9.51 \times 10^{-5}$ & $9.37 \times 10^{-7}$ & $2.98 \times 10^{-7}$ & $0.96 \times 10^{6}$ & 21.67 & 1.40 \\
\hline P2 & 1.3743 & $7.72 \times 10^{-5}$ & $7.85 \times 10^{-7}$ & $2.98 \times 10^{-7}$ & $0.93 \times 10^{6}$ & 22.05 & 1.28 \\
\hline P3 & 1.4681 & $1.81 \times 10^{-4}$ & $9.20 \times 10^{-7}$ & $7.98 \times 10^{-8}$ & $1.81 \times 10^{6}$ & 30.80 & 2.63 \\
\hline P4 & 1.8510 & $1.21 \times 10^{-4}$ & $9.45 \times 10^{-7}$ & $8.70 \times 10^{-8}$ & $1.86 \times 10^{6}$ & 29.64 & 2.73 \\
\hline P5 & 2.2858 & $2.39 \times 10^{-4}$ & $9.44 \times 10^{-7}$ & $1.58 \times 10^{-7}$ & $2.88 \times 10^{6}$ & 44.75 & 3.03 \\
\hline
\end{tabular}

speedup with respect to classical solution schemes is significant. We also elucidate complexities associated with explicit FE, in the form of a careful analysis of the mesh and material.

\subsection{Simulation workflow analysis}

\subsubsection{Pre-processing and model analysis}

The importance of a layered approach in meshing and material property assignment is apparent in Fig. 3, where the throughthickness stress distribution is significant. A single-layered approach could not resolve this distribution unless higher-order elements were used. Furthermore, these meshes are particularly suited to capture stress gradients with residual stresses included.

The challenging meshing constraints outlined in Section 1.3.2 warrant an analysis of mesh quality prior to any FE solution. Illstructured elements may yield poor results generally, as well as reduce the safe time-step, particularly in explicit solution schemes. Low time-step ensures numerical stability, while a larger step size allows less iterations to solve the BVP. Pre-processing results reveals collapsed "wedge" hexahedral elements, with zero Jacobian 

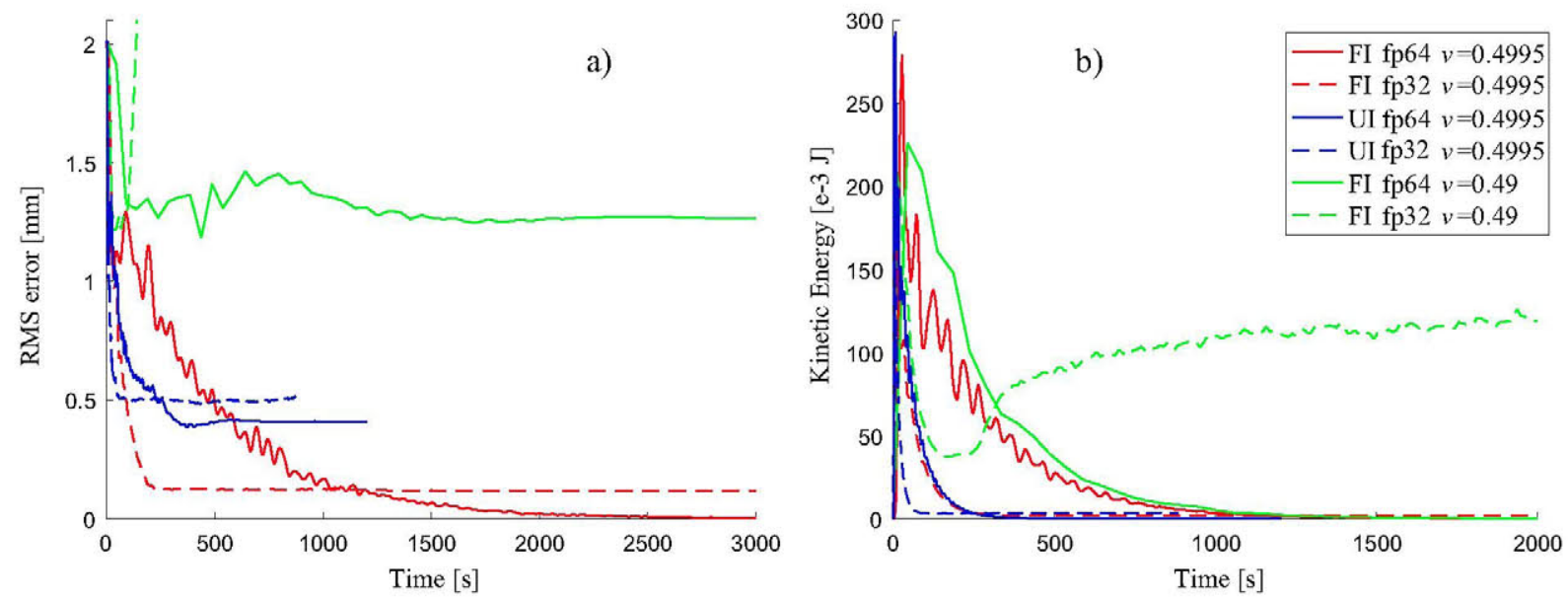

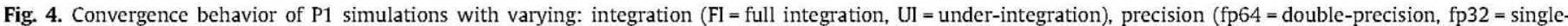

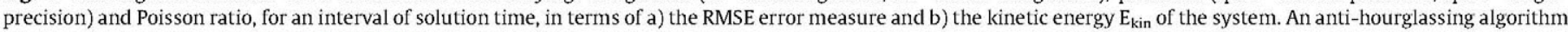
implemented according to Joldes et al. (2008) with a coefficient of 0.5 successfully counteracted the hourglass modes in the UI solutions.

Table 4

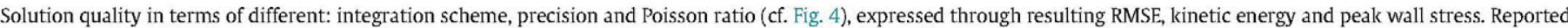
results are either at point of convergence $\left(E_{\mathrm{kn} n}<1 \times 10^{-9}\right.$ ), or at the imposed maximum step of $3 \times 10^{6}$. First row corresponds to result in Table 3 .

\begin{tabular}{|c|c|c|c|c|c|}
\hline Integration & Precision & Poisson ratio & RMSE end [mm] & $\mathrm{E}_{\mathrm{kin}}\left[10^{-3} \mathrm{~J}\right]$ & PWS [MPa] \\
\hline $\mathrm{Fl}$ & fp64 & 0.4995 & $9.51 \times 10^{-5}$ & $9.37 \times 10^{-7}$ & $9.22 \times 10^{-1}$ \\
\hline $\mathrm{Fl}$ & $\mathrm{fp} 32$ & 0.4995 & 0.12 & 1.93 & $9.38 \times 10^{-1}$ \\
\hline UI & fp64 & 0.4995 & 0.41 & $9.49 \times 10^{-7}$ & $8.14 \times 10^{-1}$ \\
\hline UI & $\mathrm{fp} 32$ & 0.4995 & 0.51 & 3.57 & $8.60 \times 10^{-1}$ \\
\hline Fl & fp64 & 0.49 & 1.26 & 7.90 & $6.32 \times 10^{-1}$ \\
\hline $\mathrm{Fl}$ & $\mathrm{fp} 32$ & 0.49 & 5.92 & 114 & $6.62 \times 10^{-1}$ \\
\hline
\end{tabular}

metric value that affect the stable time-step (cf. Table 1). The discrepancy between the projected maximum time-steps in Tables 1 and 3 is due to the conservative nature of the CFL estimate originating from large variation in element stiffnesses or sizes. Therefore, the step may be slightly increased without loss of stability. We tune the magnitude of this increase specifically for each FE model by trial and error. The stable time-step is inversely proportional to the wave speed $c$ (cf. Fig. 5(b)) which is highly sensitive to the incompressibility level, and hence the bulk modulus $K$. We set the bulk modulus to a very high value such that the initial Poisson ratio between $\mu$ and $K$ for any material layer is always $\geqslant 0.4995$. We do not include the wave speed in the table as (in the initial configuration) all patients share the same element wave speed distribution, shown in Fig. 5. Implicit methods, while unconditionally stable and easier to set up and tune by trial and error, face similar challenges in highly-nonlinear analyses.

\subsubsection{FE execution and validation}

We demonstrate significant speedup in solving state-of-the-art AAA inflation simulations. However, we draw attention to the solution scheme in terms of optimal step size and tuning. Total solution time is primarily affected by a judicious step size. As introduced in Sections 2.3.1 and 3.1, wave speed and characteristic length are the related factors. The material imposes the wave speed, while the challenging geometry limits the characteristic length. The importance of the characteristic length cannot be overstated and is perhaps best illustrated by noting the large difference in $L_{e}$ in poorlyformed and well-formed elements shown in Table 1, and the resulting effect on $\Delta t$.

At such small time steps and high material stiffness, all singleprecision results suffer from spurious displacement perturbations introduced by roundoff and truncation errors. Ultimately due to machine precision, these numerical errors affect the smoothness and accuracy of the solution, expressed through an increase in kinetic energy (cf. Table 4).

Additionally, in such conditions implicit FE is classically used to accurately solve a static model, being the faster (cf. Johnsen et al., 2015; Han et al., 2012) and more reliable method. Instead of using a similar explicit CPU solution as reference (yielding better GPU speedups) we assume the viewpoint of a novel user deciding whether a CPU or a GPU solution is preferred. Therefore, we use the faster, implicit, CPU solution as reference instead of an explicit CPU reference.

\subsection{Shortcomings in light of rupture risk assessment}

Neither CT nor MRI imaging modalities currently allow clear discrimination between individual layers of the aortic wall or thrombus. Nevertheless, since MRI can capture lumen/ILT/aortic wall separation, it allows for more precise approximation through statistically-based avenues of obtaining representative layer thicknesses (Tarjuelo-Gutierrez et al., 2014).

An important open question with such models is the lack of a non-invasive method to reliably determine patient-specific material properties (Smoljkić et al., 2015; Wittek et al., 2013). Reliable risk assessment involves the comparison of PWS to the maximum wall strength, where again, no non-invasive, non-destructive and reliable methods are established. Even with accurate information on the material parameters and maximum yield strengths, the problem of prestressing (undeformed configuration) and residual stresses remain. In light of a multitude of solutions (cf. Section 1.3.3) we focus attention on efficient forward simulation which is directly applicable in all of these contexts. 

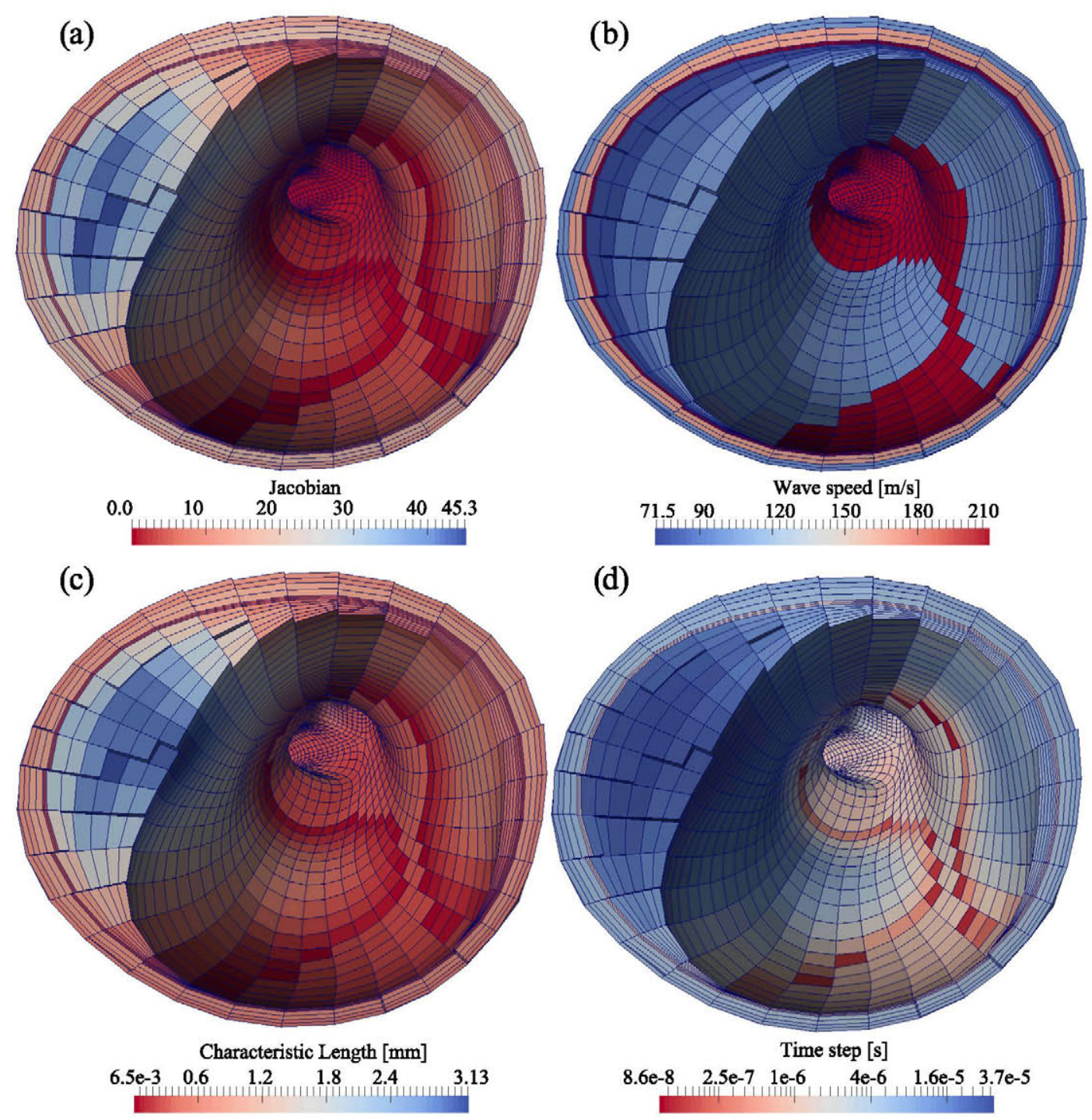

Fig. 5. Illustration of mesh quality and time-step considerations on a mesh cross-section: (a) Jacobian, (b) wave speed, (c) characteristic lengths and (d) the resulting timestep. Viewed from a transverse cross-section of P1 in the distal direction.

\subsection{Potential for further speedup}

While under-integration offers significant computational benefits generally (Strbac et al., 2016), it is presently inapplicable even with anti-hourglassing (Joldes et al., 2008; Schulz, 1985; Flanagan and Belytschko, 1981) due to strong anisotropy and collapsed elements.

Damping is a direct avenue for optimizing the method. Due to the stringent accuracy requirements, the stabilization time may take most of the solution time. The evolution of the kinetic energy and RMSE of the system converge in a typical manner for all patients, reaching approximately $95 \%$ of the solution in about half of the solution time, cf. Fig. 4. The core of the issue is the diagonal mass-proportional damping matrix (second term in Eq. (1)) which is an essential source of parallelism, indicating Dynamic Relaxation as likely an efficient remedy (Belytchko and Hughes, 1983; Joldes et al., 2009; Joldes et al., 2011) to decrease convergence time while preserving the diagonal matrix structure. Additionally, Fig. 4 shows that faster solutions may be obtained through a slight relaxation of the termination criteria.

Purely from a computational perspective, it is worth mentioning that both GPU and CPU solution approaches can be further par- allelized by using multiple GPUs as well as CPUs within a multicore or distributed computing model. While single-core performance is unrepresentative of today's conventional CPUs, estimates of multi-core performance are easily obtained through simple division of the CPU solution time, even assuming perfect scaling.

\subsection{Summary}

In summary, explicit FE, while sensitive to materials used, the geometry of elements, floating point precision and full integration, is still an excellent candidate for GPU parallelization in highly complex biomechanics problems. The decoupled nature of computation plays into the GPU computing model well, and combined with the powerful tightly-coupled hardware, offers substantial acceleration in face of significant computational difficulty (cf. Section 1.4). The clinical potential is evident: using this enabling technology, detailed biomechanical models may be used in rupture risk assessment, without the need for accuracy robbing simplifications. This is especially true given that GPU technology is advancing rapidly, and new architectures offer significant speedup without the need for re-implementation. 


\section{Conflict of interest}

The authors have no conflicts of interest to declare.

\section{Acknowledgments}

This research was supported by the Research FoundationFlanders (G.0.932.11 and PDO/12) and the European Commission's 7th Framework Programme FP7-ICT - under grant agreement No. 601021 (CASCADE).

\section{Appendix A. Supplementary material}

Supplementary data associated with this article can be found, in the online version, at http://dx.doi.org/10.1016/j.jbiomech.2017. 02.019 .

\section{References}

Alastrué, V., Garía, A., Peña, E., Rodríguez, J.F., Martínez, M.A., Doblaré, M., 2010. Assessing the use of the "opening angle method" to enforce residual atresses in patient-specific arteries. Int. J. Numer. Meth. Biomed. Eng. 26, 35-51.

Alastrué, V., Peña, E., Martínez, M., Doblaré, M., 2007. Assessing the use of the "opening angle method" to enforce residual atresses in patient-specific arteries. Ann. Biomed. Eng. 35, 1821-1837.

Auer, M., Gasser, T.C., 2010. Reconstruction and finite element mesh generation of abdominal aortic aneurysms from computerized tomography angiography data with minimal user interactions. IEEE Trans. Med. Imag. 29, 1022-1028.

Bartezzaghi, A., Cremonesi, M., Parolini, N., Perogo, U., 2015. An explicit dynamics GPU structural solver for thin shell finite elements. Comput. Struct. 154, 29-40.

Bellini, C., Ferruzzi, J., Roccabianca, S., Di Martino, E.S., Humphrey, J.D., 2014. A microstructurally motivated model of arterial wall mechanics with mechanobiological implications. Ann. Biomed. Eng. 42, 488-502.

Belytchko, T., Hughes, T.J.R. (Eds.), 1983. Computational Methods for Transient Analysis. North-Holland, Amsterdam, Netherlands.

Belytschko, T., 1976. A survey of numerical methods and computer programs for dynamic structural analysis. Nucl. Eng. Des. 31, 23-34.

Belytschko, T., Liu, W.K., Moran, B., 2000. Nonlinear Finite Elements for Continua and Structures. John Wiley \& Sons, Ltd., West Sussex, UK.

Bols, J., Degroote, J., Trachet, B., Verhegghe, B., Segers, P., Vierendeels, J., 2013. A computational method to assess the in vivo stresses and unloaded configuration of patient-specific blood vessels. J. Comput. Appl. Math. 246, 10-17.

Cecka, C., Lew, A.J., Darve, E., 2011. Assembly of finite element methods on graphics processors. Int. J. Numer. Meth. Eng. 85, 640-669.

Chaikof, E.L., Brewster, D.C., Dalman, R.L., Makaroun, M.S., Illig, K.A., Sicard, G.A., Timaran, C.H., Upchurch Jr., G.R., Veith, F.J., 2009. The care of patients with an abdominal aortic aneurysm: the society for Vascular Surgery practice guidelines. J. Vasc. Surg. 50, S2-S49.

Comas, O., Taylor, Z.A., Allard, J., Ourselin, S., Cotin, S., Passenger, J., 2008. Efficient nonlinear fem for soft tissue modelling and its GPU implementation within the open source framework SOFA. In: Bello, F., Edwards, P.J. (Eds.), Biomedical Simulation, Lecture Notes in Computer Science, vol. 5104. Springer, Berlin, Heidelberg, pp. 28-39.

Courant, R., Friedrichs, K., Lewy, H., 1967. On the partial difference equations of mathematical physics. IBM J. Res. Develop. 11, 215-234.

de Putter, S., Wolters, B.J.B.M., Rutten, M.C.M., Breeuwer, M., Gerritsen, F.A., van de Vosse, F.N., 2007. Patient-specific initial wall stress in abdominal aortic aneurysms with a backward incremental method. J. Biomech. 40, 1081-1090.

De Santis, G., De Beule, M., Van Canneyt, K., Segers, P., Verdonck, P., Verhegghe, B., 2011. Full-hexahedral structured meshing for image-based computational vascular modeling. Med. Eng. Phys. 33, 1318-1325.

Fillinger, M.F., Raghavan, M.L., Marra, S.P., Cronenwett, J.L., Kennedy, F.E., 2002. In vivo analysis of mechanical wall stress and abdominal aortic aneurysm rupture risk. J. Vasc. Surg. 36, 589-597.

Flanagan, D.P., Belytschko, T., 1981. A uniform strain hexahedron and quadrilateral with orthogonal hourglass control. Int. J. Numer. Meth. Eng. 17, 679-706.

Fleming, C., Whitlock, E.P., Beil, T.L., Lederle, F.A., 2005. Screening for abdominal aortic aneurysm: a best-evidence systematic review for the U.S. Preventive Services Task Force. Ann. Int. Med. 142, 203-211.

Gasser, T.C., Auer, M., Labruto, F., Swedenborg, J., Roy, J., 2010. Biomechanical rupture risk assessment of abdominal aortic aneurysms: model complexity versus predictability of finite element simulations. Eur. J. Vasc. Endovasc. Surg. 40, $176-185$.

Gasser, T.C., Ogden, R.W., Holzapfel, G.A., 2006. Hyperelastic modelling of arterial layers with distributed collagen fibre orientations. J. R. Soc. Interface 3, 15-35.

Grandy, J., 1997. Efficient Computation of Volume of Hexahedral Cells Technical Report UCRL-ID-128886. Lawrence Livermore National Laboratory.

Han, L., Hipwell, J., Taylor, Z., Tanner, C., Ourselin, S., Hawkes, D.J., 2010. Fast deformation simulation of breasts using gpu-based dynamic explicit finite element method. In: Martí, J., Arnau, O., Freixenet, J., Martí, R. (Eds.), Digital
Mammography: 10th International Workshop, IWDM 2010, Girona, Catalonia, Spain, June 16-18, 2010. Proceedings. Springer, Berlin, Heidelberg, pp. 728-735.

Han, L., Hipwell, J.H., Tanner, C., Mertzanidou, T., Cardoso, J., Ourselin, S., Hawkes, D. J., 2012. Development of patient-specific biomechanical models for predicting large breast deformation. Phys. Med. Biol. 57, 455-472.

Harris, M., 2013. Optimizing Parallel Reduction in CUDA. Nvidia Corporation.

Helfenstein, J., Jabareen, M., Mazza, E., Govindjee, S., 2010. On non-physical response in models for fiber-reinforced hyperelastic materials. Int. J. Solids Struct. 47, 2056-2061.

Holzapfel, G.A., Gasser, T.C., Ogden, R.W., 2000. A new constitutive framework for arterial wall mechanics and a comparative study of material models. J. Elast. 61 $1-48$.

Holzapfel, G.A., Sommer, G., Gasser, C.T., Regitnig, P., 2005. Determination of layerspecific mechanical properties of human coronary arteries with nonatherosclerotic intimal thickening and related constitutive modeling. Am. J. Physiol. Heart Circ. Physiol. 289, H2048-H2058.

Huthwaite, P., 2014. Accelerated finite element elastodynamic simulations using the GPU. J. Comput. Phys. 257, 687-707.

Johansson, G., Nydahl, S., Olofsson, P., Swedenborg, J., 1990. Survival in patients with abdominal aortic aneurysms. Comparison between operative and nonoperative management. Eur. J. Vasc. Surg. 4, 497-502.

Johnsen, S.F., Taylor, Z.A., Clarkson, M.J., Hipwell, J., Modat, M., Eiben, B., Han, L., Hu, Y., Mertzanidou, T., Hawkes, D.J., Ourselin, S., 2015. Niftysim: a GPU-based nonlinear finite element package for simulation of soft tissue biomechanics. Int. J. Comput. Assist. Radiol. Surg. 10, 1077-1095.

Joldes, G.R. Wittek, A. Miller, K, 2008. An efficient hourglass control implementation for the uniform strain hexahedron using the Total Lagrangian formulation. Commun. Numer. Meth. Eng. 24, 1315-1323.

Joldes, G.R., Wittek, A., Miller, K., 2009. Computation of intra-operative brain shift using dynamic relaxation. Comput. Meth. Appl. Mech. Eng. 198, 3313 3320 .

Joldes, G.R., Wittek, A., Miller, K., 2010. Real-time nonlinear finite element computations on GPU - application to neurosurgical simulation. Comput. Meth. Appl. Mech. Eng. 199, 3305-3314.

Joldes, G.R., Wittek, A., Miller, K., 2011. An adaptive Dynamic Relaxation method for solving nonlinear finite element problems. Application to brain shift estimation. Int. J. Numer. Meth. Biomed. Eng. 27, 173-185.

Joldes, G.R., Wittek, A., Miller, K., 2015. A Total Lagrangian based method for recovering the un-deformed configuration in finite elasticity. Appl. Math Model, 39, 3913-3923.

Martufi, G., Forneris, A., Apoo, J.J., Di Martino, E.S., 2016. Is there a role for biomechanical engineering in helping to elucidate the risk profile of the thoracic aorta? Ann. Thorac. Surg. 101, 390-398.

Miller, K., Joldes, G.R., Lance, D., Wittek, A., 2007. Total Lagrangian explicit dynamics finite element algorithm for computing soft tissue deformation. Commun. Numer. Meth. Eng. 23, 121-134.

Nvidia Corp., 2015. CUDA C Programming Guide v7.5. Technical Report. Nvidia Corporation.

Pierce, D.M., Fastl, T.E., Rodriguez-Vila, B., Verbrugghe, P., Fourneau, I., Maleux G., Herijgers, P., Gomez, E.J., Holzapfel, G.A., 2015a. A method for incorporating three-dimensional residual stretches/stresses into patientspecific finite element simulations of arteries. J. Mech. Behav. Biomed. Mater. 47, 147-164.

Pierce, D.M. Maier, $F$, Weisbecker, $H$, Viertler, C, Verbrugghe, P., Famaey, N., Fourneau, I., Herijgers, P., Holzapfel, G.A., 2015b. Human thoracic anc abdominal aortic aneurysmal tissues: damage experiments, statistical analysis and constitutive modeling. J. Mech. Behav. Biomed. Mater. 41, 92-107.

Puso, M.A., Solberg, J., 2011. A stabilized nodally integrated tetrahedral. Int. J. Numer. Meth. Eng. 67, 841-867.

Rasmusson, A., Mosegaard, J., Sørensen, T.S., 2008. Exploring parallel algorithms for volumetric mass-spring-damper models in CUDA. In: Bello, F., Edwards, P.J (Eds.), Biomedical Simulation, Lecture Notes in Computer Science, vol. 5104 Springer, Berlin, Heidelberg, pp. 49-58.

Schulz, J.C., 1985. Finite element hourglassing control. Int. J. Numer. Meth. Eng. 21, 1039-1048.

Smistad, E., Falch, T.L., Bozorgi, M., Elster, A.C., Lindseth, F., 2015. Medical image segmentation on GPUs - a comprehensive review. Med. Image Anal. 20, 1-18.

Smoljkić, M., Sloten, J.V., Segers, P., Famaey, N., 2015. Non-invasive, energy-based assessment of patient-specific material properties of arterial tissue. Biomech. Model. Mechanobiol. 14, 1045-1056.

Stimpson, C.J., Ernst, C.D., Knupp, P., Pébay, P.P., Thompson, D., 2007. The Verdict Geometric Quality Library Technical Report SAND2007-1751. Sandia National Laboratories.

Strbac, V., Pierce, D.M., Sloten, J.V., Famaey, N., 2016. Gpu-based fast finite element solution for nonlinear anisotropic material behavior and comparison of integration strategies. In: Joldes, G.R., Doyle, B., Wittek, A., Nielsen, P.M.F., Miller, K. (Eds.), Computational Biomechanics for Medicine: Imaging, Modeling and Computing. Springer International Publishing, pp. 97-105.

Strbac, V., Vander Sloten, J., Famaey, N., 2015. Analyzing the potential of GPGPUs for real-time explicit finite element analysis of soft tissue deformation using CUDA. Finite Elem. Anal. Des. 105, 79-89.

Tarjuelo-Gutierrez, J., Rodriguez-Vila, B., Pierce, D.M., Fastl, T.E., Verbrugghe, P. Fourneau, I., Maleux, G., Herijgers, P., Holzapfel, G.A., Gomez, E.J., 2014. Highquality conforming hexahedral meshes of patient-specific abdominal aortic aneurysms including their intraluminal thrombi. Med. Biol. Eng. Comput. 52, $159-168$. 
Taylor, Z.A., Comas, O., Cheng, M., Passenger, J., Hawkes, D.J., Atkinson, D., Ourselin, S., 2009. On modelling of anisotropic viscoleasticity for soft tissue simulation: numerical solution and GPU execution. MEDIA 13, 234-244.

Tong, J., Cohnert, T., Regitnig, P., Holzapfel, G.A., 2011. Effects of age on the elastic properties of the intraluminal thrombus and the thrombus-covered wall in abdominal aortic aneurysms: biaxial extension behaviour and material modelling. Eur. J. Vasc. Endovasc. Surg. 42, 207-219.

Vardulaki, K.A., Prevost, T.C., Walker, N.M., Day, N.E., Wilmink, A.B.M., Quick, C.R.G Ashton, H.A., Scott, R.A.P., 1998. Growth rates and risk of rupture of abdominal aortic aneurysms. Brit. J. Surg. 85, 1674-1680.

Weisbecker, H., Pierce, D.M., Holzapfel, G.A., 2014. A generalized prestressing algorithm for finite element simulations. Int. J. Numer. Meth. Biomed. Eng. 30 857-872.
Weisbecker, H., Pierce, D.M., Regitnig, P., Holzapfel, G.A., 2012. Layer-specific damage experiments and modeling of human thoracic and abdominal aortas with non-atherosclerotic intimal thickening. J. Mech. Behav. Biomed. Mater. 12, 93-106.

Wittek, A., Karatolios, K., Bihari, P., Schmitz-Rixen, T., Moosdorf, R., Vogt, S., Blase, C. 2013. In vivo determination of elastic properties of the human aorta based on 4D ultrasound data. J. Mech. Behav. Biomed. Mater. 27, 167-183.

Wong, J., Kuhl, E., Darve, E., 2015. A new sparse matrix vector multiplication graphics processing unit algorithm designed for finite element problems. Int. J. Numer. Meth. Eng. 102, 1784-1814. 